\title{
A Method to Detect Pauses for Ventilation During Cardiopulmonary Resuscitation Using the Thoracic Impedance
}

\author{
Enrique Rueda ${ }^{1}$, Elisabete Aramendi ${ }^{1}$, Unai Irusta ${ }^{1}$, Ahamed H. Idris ${ }^{2}$ \\ ${ }^{1}$ Biores, University of the Basque Country, UPV/EHU Bilbao, Spain \\ ${ }^{2}$ University of Texas Southwestern Medical Center (UTSW), Dallas, Texas
}

\begin{abstract}
Cardiac arrest is the main cause of death in developed countries. A good quality cardiopulmonary resuscitation (CPR) is key for the survival of the patient in outof-hospital cardiac arrest (OHCA), including chest compressions (CCs) and ventilations. Ventilations have been proven to have an important impact in the outcome of the patient, and detecting the CC pauses where ventilations were provided is the aim of this study. An algorithm that automatically detects pauses between sequences of CCs using machine learning techniques is proposed.

For this study a set of 102 defibrillator files from $\mathrm{OHCA}$ patients that include the thoracic impedance recorded through the defibrillation pads was used. The work has been split into 2 main blocks: a random forest $(R F)$ classifier that classifies 1-s windows as CC/no-CC and an algorithm that sets the beginning and the end of each detected pause. The RF classifier was validated using 10 fold cross-validation method, obtaining a median sensitivity (Se), specificity $(S p)$ and positive predictive value (PPV) of 95.4/97.9/94.4\% respectively, for window classification. The pause detector returned median Se/PPV values of 90.0/91.3\% with a median pause delimitation error of $0.04 \mathrm{~s}$ and a duration error of $0.04 \mathrm{~s}$.
\end{abstract}

\section{Introduction}

Sudden cardiac death is the main death cause in developed countries, with an incidence of 38 cases per 100000 person-year in Europe [1]. Although progress has been made in different fields, survival rates in out-of-hospital cardiac arrest (OHCA) remains close to $12 \%$ [2].

Cardiopulmonary resuscitation (CPR) is the key treatment for OHCA. According to the latest resuscitation guidelines, CPR can be performed using continuous chest compression or by a combination of chest compressions (CCs) and ventilations with a rate of 30-2 [3]. Several studies have proven the benefits of effective ventilations during CC-pauses which are provided before the patient is intubated $[3,4]$. In that period only the thoracic impedance (TI) acquired through defibrillation pads is available. This signal can be used to monitor CPR because it exhibits fluctuations due to both CCs and ventilations [5].

As an initial step in the monitoring of ventilations during advanced airway management in 30-2 protocol, this study proposes an algorithm based on machine learning techniques to detect CC-pauses in the TI.

\section{Materials}

The data consists of 102 defibrillator files from OHCA cases that occurred in the area of Dallas Fort-Worth between January 2015 and December 2016. These episodes were recorded by the DFW Center for Resuscitation Research (UTSW, Dallas) using LifePak 12 and 15 monitor/defibrillators. Each record included the ECG and the TI signals, where CCs and pauses between CCs were manually annotated to be used as gold standard in the algorithm development.

In total, 1936 minutes of recordings corresponding to the pre-intubation phase were processed, with $194 \mathrm{~min}$ utes of pauses with a median (Q1-Q3) duration of 14 (10$25)$ min and 13 (9-19) pauses per patient with a duration of $6.06(4.65-8.19) \mathrm{s}$.

\section{Methods}

TI signal was first filtered using third order Butterworth passband filter $(0.9-6 \mathrm{~Hz})$ and forward-backward filtering to remove high frequency noise and baseline wandering. The signal was divided into 1-s windows, which were classified as CC/no-CC window using a Random Forest (RF) classifier. The RF used 18 waveform features for classification, computed in the time, frequency, complexity and statistical domains. The classifier was trained using 10fold cross validation method, which was used also to perform a feature selection. Finally, a simple algorithm was used to delimit the pauses based on the classification given by the RF classifier. Pauses with a duration of 3-15 s were detected as pauses associated to ventilations. The top panel 

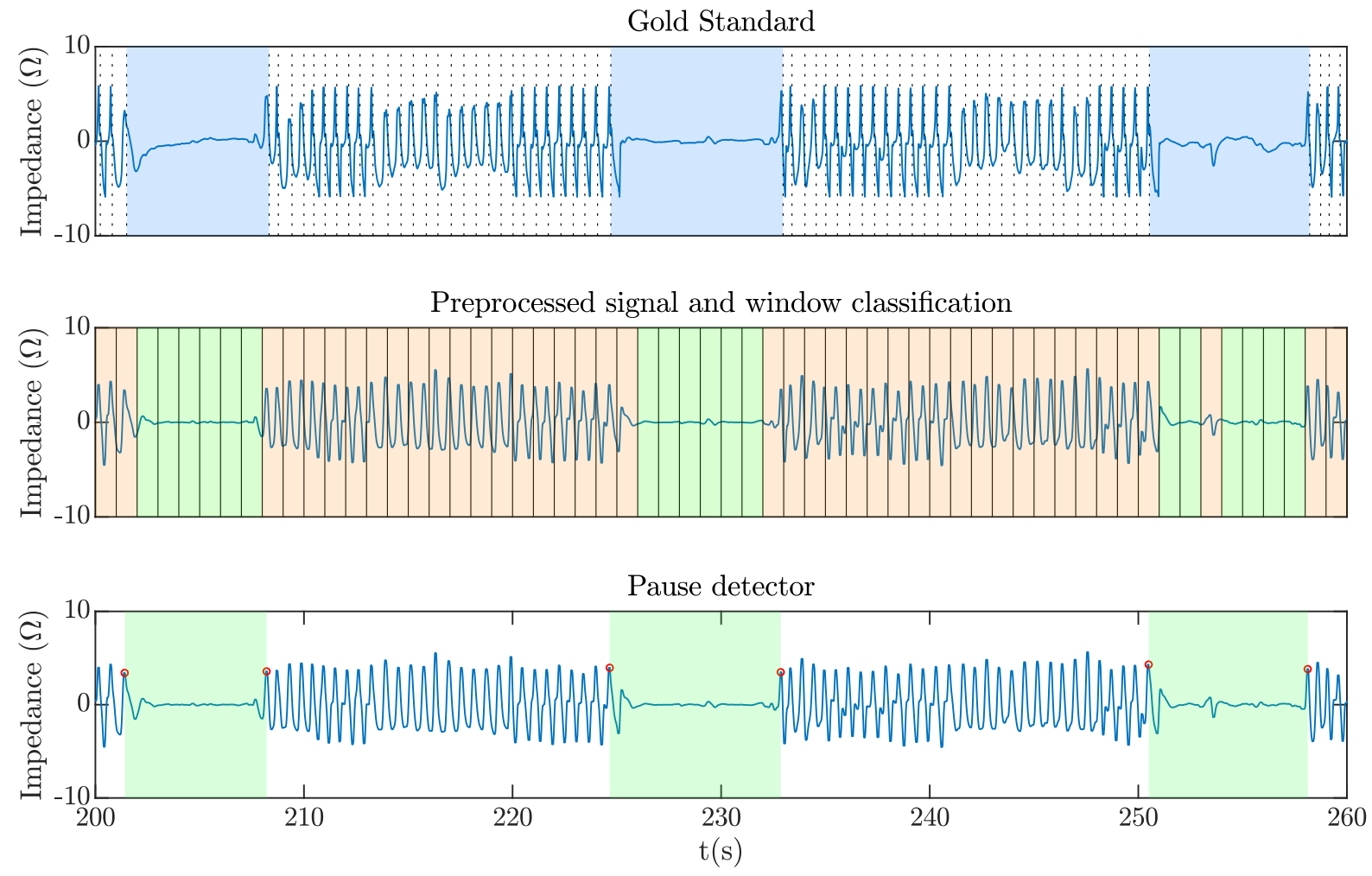

Figure 1. A 60 seconds example of an episode's TI signal. In the picture above the original signal is drawn along with chest-compression marks and a Gold Standard pause in blue. In the picture in the middle the processed signal with 1 second windows can be found. Windows in orange belong to windows classified as CC. On the other hand, windows in green correspond to windows classified as no-CC. In the picture below, pauses detected by the pause detector can be found over the processed signal. Red circles mark the last and first compressions detected at the beginning and end of each pause.

of Figure 1 shows an interval of $60 \mathrm{~s}$ of the TI, where CCs were annotated by expert clinicians, and delimited pauses (in blue) were used as gold standard for the algorithm design and validation.

\subsection{Machine Learning classifier}

The classification was made for every 1-s windows of the preprocessed TI signal (s1). The set of 18 features that were computed for each window is summarized in Table 1. These features can be classified in 4 domains: time domain (D1), frequency domain (D2), statistical domain (D3) and complexity domain (D4). Features $f_{1}$ and $f_{11}$ are the logarithm of summation of the absolute correlations (LAC) of the autocorrelation and the cross-correlation with the previous segment, respectively. Features $f_{2}$ and $f_{12}$ are the variance of the peaks of the autocorrelation and the crosscorrelation with the previous segment, respectively. Features $f_{3}$ and $f_{4}$ are the percentage of power in the $1-3 \mathrm{~Hz}$ bandwidth and the peak frequency [6]. Feature $f_{5}$ is de- fined as the summation of the absolute amplitude of the first difference of the signal. To calculate $f_{6}$ each window is divided into 3 windows, and the median value of the standard deviation (std) of the window is computed [6]. Features $f_{7}$ and $f_{8}$ are the statistical parameters skewness and kurtosis of the amplitude distributions. The corrected conditional entropy and the permutation entropy of the signal, as described in [7] and [8], are computed as $f_{9}$ and $f_{10}$. Features $f_{13-16}$ are computed as $f_{1}, f_{5}, f_{4}$ and $f_{9}$ respectively, for the high-pass $(2-8 \mathrm{~Hz})$ filtered TI signal (s2). The last 2 features, $f_{17}$ and $f_{18}$, are the relative variation of the window signal amplitude and slope compared to the average of previous 3 segments.

The RF is based on bagging concept: $\mathrm{T}$ decision trees are trained to produce $\mathrm{T}$ uncorrelated predictions and the final decision is made by majority voting [9]. Uncorrelated decisions are made by using different bootstraps of training data to train each tree, and also by using a limited set of randomly selected features in each tree split. The RF classifier allows also to measure the importance of each feature 
Table 1. List of extracted features.

\begin{tabular}{lll}
\hline \hline$f_{1}$ & LAC of autocorrelation & D1 \\
$f_{2}$ & variance of autocorrelation peaks & D1 \\
$f_{3}$ & power in 1-3 Hz bandwidth & D2 \\
$f_{4}$ & peak frequency & D2 \\
$f_{5}$ & standard deviation of amplitude & D1 \\
$f_{6}$ & summation of slope & D1 \\
$f_{7}$ & skewness & D3 \\
$f_{8}$ & kurtosis & D3 \\
$f_{9}$ & corrected conditional entropy & D4 \\
$f_{10}$ & permutation entropy & D4 \\
$f_{11}$ & LAC of cross-correlation & D1 \\
$f_{12}$ & variance of cross-correlation peaks & D1 \\
$f_{13}$ & LAC of s2 & D1 \\
$f_{14}$ & slope of s2 & D1 \\
$f_{15}$ & frequency of peak power in s2 & D2 \\
$f_{16}$ & corrected conditional entropy of s2 & D4 \\
$f_{17}$ & relative amplitude & D1 \\
$f_{18}$ & relative slope & D1 \\
\hline \hline
\end{tabular}

by permuting the value of each feature and measuring the out-of-bag error (error produced using the data that each tree did not see during training process). This functionality was used to perform feature selection, by taking out the least important feature.

\subsection{Pause delineation}

The RF classifier's outcome is a binary classification of CC/no-CC for each window. Two consecutive non CC windows were considered as a potential CC-pause, and the first and last $\mathrm{CC}$ of the pause, which set the beginning and end of it, were detected using a peak detector. Pauses with a duration of 3-15 s were thus identified. The lowest panel in Figure 1 shows the pauses detected by the algorithm in an example of the dataset.

\subsection{Model assessment}

Model validation was handled by 10 -fold crossvalidation method. Backward feature elimination was used to adjust model with different numbers of features. At each training iteration a RF classifier was trained, feature importance was measured, and the least important feature was removed. The process was repeated until a single feature was left. At each step, results were obtained for the test fold and performance was thus obtained as a function of the number of features $(N)$ in the model. .

The performance of the RF classifier was characterized using the sensitivity (Se) to detect no-CC windows, speci- ficity ( $\mathrm{Sp}$ ) to correctly detect $\mathrm{CC}$ windows, positive predictive value (PPV), $\mathrm{F}_{1}$-score and area under the receiver operating characteristics curve (AUC).The CC-pause detector was evaluated using Se and PPV. Absolute error in delineation of the start of the pause and its duration were also given. Every metric was reported as median quartile range $\mathrm{Q} 1-\mathrm{Q} 3$ values.

\section{Results}

This section shows the results of the RF classifier and the pause detector.

\subsection{Random Forest classifier}

Figures 2 and 3 shows the median (Q1-Q3) values of the AUC and $F_{1}$ for different number of features of the model $(\mathrm{N})$. It can be observed that for $\mathrm{N}$ above 10 little increase of performance is obtained. The selected $\mathrm{N}$ features were: $f_{l}$, $f_{3}, f_{4}, f_{5}, f_{6}, f_{8}, f_{10}, f_{11}, f_{17}$ and $f_{18}$. Features $f_{13-16}$ were considered redundant by the classifier and were not selected by the RF.

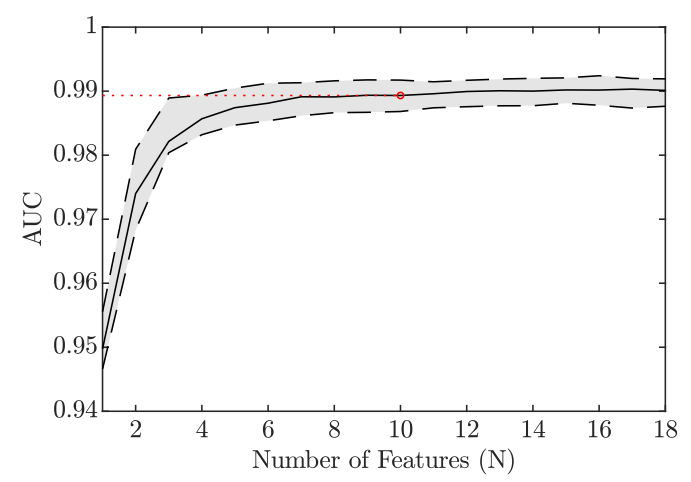

Figure 2. Median and Q1-Q3 range of the AUC values of the RF classifier in terms of the number of features.

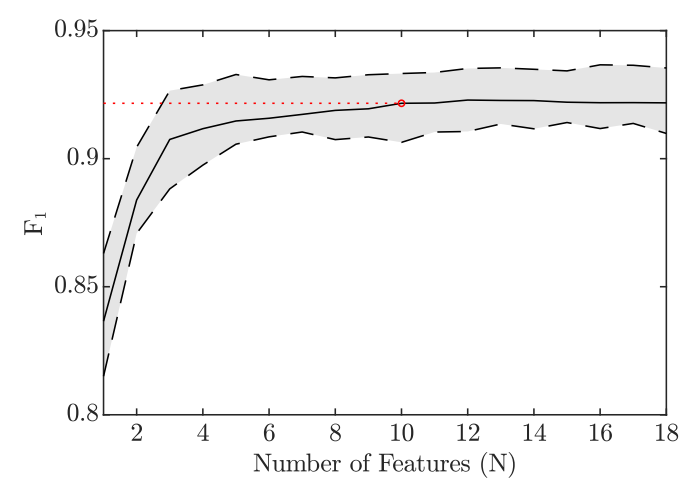

Figure 3. Median and Q1-Q3 range $F_{1}$ values of the RF classifier in terms of the number of features. 
The performance metrics for the RF classifier are given in Table 1 for both $\mathrm{N}=10$ and all features. In this case, the most selected 10 features were used in a 10 -fold crossvalidation procedure.

Table 2. Median (Q1-Q3) of the RF performance metrics.

\begin{tabular}{lcc}
\hline & $\mathrm{N}=10$ & $\mathrm{~N}=18$ \\
\hline Se (\%) & $95.4(91.3-97.9)$ & $95.7(91.6-98.0)$ \\
Sp (\%) & $97.9(96.2-98.9)$ & $97.7(95.9-99.0)$ \\
PPV (\%) & $94.4(90.8-97.4)$ & $95.1(91.4-97.6)$ \\
AUC & $0.989(0.987-0.992)$ & $0.990(0.988-0.992)$ \\
\hline
\end{tabular}

\subsection{Pause detector}

The Se and PPV values for the pause detector were $90.0 / 91.3 \%$, with a median error at pause start of the detected pauses was $0.04(0.002-0.72) \mathrm{s}$ and median error of $0.04(0.01-1.34) \mathrm{s}$ in measuring the duration of the pause.

\section{Conclusions}

The proposed method for pause detection was designed and tested using the TI signal recorded while treating OHCA patients. Two algorithms were evaluated, a RF classifier that classifies 1-s windows of TI signal as CC/no$\mathrm{CC}$, and the pause detector. For the first one the classifier provided a median Se/Sp/PPV of 95.4/97.9/94.4\% with an AUC of 0.989 . The final CC pause detector produced a $\mathrm{Se} / \mathrm{PPV}$ of $90.0 / 91.3 \%$ with a delimitation error of $0.04 \mathrm{~s}$ at pause start, and a median pause duration error of $0.04 \mathrm{~s}$.

\section{Acknowledgements}

This work was supported by the Spanish Ministerio de Ciencia, Innovacion y Universidades through grant RTI2018-101475-BI00, jointly with the Fondo Europeo de Desarrollo Regional (FEDER), and by the Basque Government through grants IT1229-19 and PRE_2019_0262.

\section{References}

[1] Atwood C, Eisenberg MS, Herlitz J, Rea TD. Incidence of ems-treated out-of-hospital cardiac arrest in europe. Resuscitation 2005;67(1):75-80.

[2] Mozaffarian D, Benjamin EJ, Go AS, Arnett DK, Blaha MJ, Cushman M, Das SR, De Ferranti S, Després JP, Fullerton HJ, et al. Executive summary: heart disease and stroke statistics-2016 update: a report from the american heart association. Circulation 2016;133(4):447-454.

[3] Perkins GD, Handley AJ, Koster RW, Castrén M, Smyth MA, Olasveengen T, Monsieurs KG, Raffay V, Gräsner JT, Wenzel V, et al. European resuscitation council guidelines for resuscitation 2015: Section 2. adult basic life support and automated external defibrillation. Resuscitation 2015;95:8199.

[4] Chang MP, Lu Y, Leroux B, Ecenarro EA, Owens P, Wang HE, Idris AH. Association of ventilation with outcomes from out-of-hospital cardiac arrest. Resuscitation 2019;141:174181.

[5] Aramendi E, Lu Y, Chang MP, Elola A, Irusta U, Owens P, Idris AH. A novel technique to assess the quality of ventilation during pre-hospital cardiopulmonary resuscitation. Resuscitation 2018;132:41-46.

[6] Coult J, Blackwood J, Rea TD, Kudenchuk PJ, Kwok H. A method to detect presence of chest compressions during resuscitation using transthoracic impedance. IEEE Journal of Biomedical and Health Informatics 2019;24(3):768-774.

[7] Porta A, Baselli G, Liberati D, Montano N, Cogliati C, Gnecchi-Ruscone T, Malliani A, Cerutti S. Measuring regularity by means of a corrected conditional entropy in sympathetic outflow. Biological Cybernetics 1998;78(1):71-78.

[8] Bandt C, Pompe B. Permutation entropy: a natural complexity measure for time series. Physical Review Letters 2002; 88(17):174102.

[9] Breiman L. Random forests. Machine Learning 2001; 45(1):5-32.

Address for correspondence:

Enrique Rueda

Engineering School of Bilbao

enrique.rueda@ehu.eus 